\title{
An efficient linear method for the estimation of ego-motion from optical flow
}

\author{
Florian Raudies and Heiko Neumann \\ Institute of Neural Information Processing \\ University of Ulm \\ 89069 Ulm, Germany
}

\begin{abstract}
Approaches to visual navigation, e.g. used in robotics, require computationally efficient, numerically stable, and robust methods for the estimation of ego-motion. One of the main problems for egomotion estimation is the segregation of the translational and rotational component of ego-motion in order to utilize the translation component, e.g. for computing spatial navigation direction. Most of the existing methods solve this segregation task by means of formulating a nonlinear optimization problem. One exception is the subspace method, a wellknown linear method, which applies a computationally high-cost singular value decomposition (SVD). In order to be computationally efficient a novel linear method for the segregation of translation and rotation is introduced. For robust estimation of ego-motion the new method is integrated into the Random Sample Consensus (RANSAC) algorithm. Different scenarios show perspectives of the new method compared to existing approaches.
\end{abstract}

\section{Motivation}

For many applications visual navigation and ego-motion estimation is of prime importance. Here, processing starts with the estimation of optical flow using a monocular spatio-temporal image sequences as input followed by the estimation of ego-motion.

Optical flow fields generated by ego-motion of the observer are getting more complex if one or multiple objects move independently of ego-motion. A challenging task is to segregate such moving objects (IMOs), where MacLean et al. proposed a combination of ego-motion estimation and the Expectation Maximization (EM) algorithm [15]. With this algorithm a single motion model is estimated for ego-motion and each IMO using the subspace method [9]. A key functionality of the subspace method is the possibility to cluster ego-motion and motion of IMOs. More robust approaches assume noisy flow estimates besides IMOs when estimating ego-motion with the EM algorithm [16,5]. Generally, the EM algorithm uses an iterative computational scheme and in each iteration the evaluation of the method estimating ego-motion is required. This necessitates a computationally highly efficient algorithm for the estimation of ego-motion in 
real-time applications. So far, many of the ego-motion algorithms introduced in the past lack this property of computationally efficiency.

Bruss and Horn derived a bilinear constraint to estimate ego-motion by utilizing a quadratic Euclidian metric to calculate errors between input flow and model flow [3]. The method is linear w.r.t. either translation or rotation and independent of depth. This bilinear constraint was used throughout the last two decades for ego-motion estimation: (i) Heeger and Jepson built their subspace method upon this bilinear constraint [9]. (ii) Chiuso et al. used a fix-point iteration to optimize between rotation (based on the bilinear constraint), depth, and translation, [4] and Pauwels and Van Hulle used the same iteration mechanism optimizing for rotation and translation (both based on the bilinear constraint) [16]. (iii) Zhang and Tomasi as well as Pauwels and Van Hulle used a GaussNewton iteration between rotation, depth, and translation [20,17]. In detail the method (i) needs singular value decomposition, and methods of (ii) and (iii) iterative optimization techniques.

Here, a novel linear approach for the estimation of ego-motion is presented. Our approach utilizes the bilinear constraint, the basis of many nonlinear methods. Unlike to these previous methods, here, a linear formulation is achieved by introducing auxiliary variables. In turn with this linear formulation a computationally efficient method is defined.

Section 2 gives a formal description of the instantaneous optical flow model. This model serves as basis to derive our method outlined in Section 3. An evaluation of the new method in different scenarios and in comparison to existing approaches is given in Section 4. Finally, Section 5 discusses our method in the context of existing approaches $[3,9,11,20,16,18]$ and Section 6 gives a conclusion.

\section{Model of instantaneous ego-motion}

Von Helmholtz and Gibson introduced the definition of optical flow as moving patterns of light falling upon the retina $[10,8]$. Following this definition LonguetHiggins and Prazdny gave a formal description of optical flow which is based on a model of instantaneous ego-motion [13]. In their description they used a pinhole camera with the focal length $f$ which projects 3 -d points $(X, Y, Z)$ onto the 2 -d image plane, formally $(x, y)=f / Z \cdot(X, Y)$. Ego-motion composed of the translation $T=\left(t_{x}, t_{y}, t_{z}\right)^{t}$ and rotation $R=\left(r_{x}, r_{y}, r_{z}\right)^{t}$ causes the 3 -d instantaneous

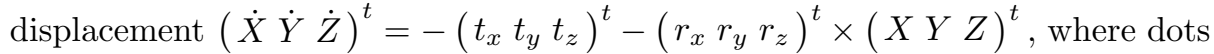
denote the first temporal derivative and ${ }^{t}$ the transpose operator. Using this model, movements of projected points on the 2-d image plane have the velocity

$$
V:=\left(\begin{array}{l}
u \\
v
\end{array}\right)=\frac{1}{Z}\left(\begin{array}{ccc}
-f & 0 & x \\
0 & -f & y
\end{array}\right) T+\frac{1}{f}\left(\begin{array}{ccc}
x y & -\left(f^{2}+x^{2}\right) & f y \\
\left(f^{2}+y^{2}\right) & -x y & -f x
\end{array}\right) R .
$$




\section{Linear method for ego-motion estimation}

Input flow, e.g. estimated from a spatio-temporal image sequence, is denoted by $\hat{V}$, while the model flow is defined as in Equation 1. Now, the problem is to find parameters of the model flow which describe the given flow $\hat{V}$ best. Namely, these parameters are the scenic depth $Z$, the translation $T$ and rotation $R$. Based on Equation 1 many researchers studied non-linear optimization problems to estimate ego-motion $[3,20,4,18]$. Moreover, most of these methods have a statistical bias which means that methods produce systematic errors considering isotropic noisy input $[14,16]$. Unlike these approaches we suggest a new linearized form based on Equation 1 and show how to solve this form computationally efficient with a new method. Further, this method can be unbiased. The new method is derived in three consecutive steps: (i) the algebraic transformation of Equation 1 which is independent of depth $Z$, (ii) a formulation of an optimization problem for translation and auxiliary variables, and (iii) the removal of a statistical bias. The calculation of rotation $R$ with translation $T$ known is then a simple problem.

Depth independent constraint equation. Bruss and Horn formulated an optimization problem with respect to depth $Z$ which optimizes the squared Euclidian distance of the residual vector between the input flow vector $\hat{V}=$ $(\hat{u}, \hat{v})^{t}$ and the model flow vector $V$ defined in Equation 1. Inserting the optimized depth into Equation 1 they derived the so called bilinear optimization constraint. An algebraic transformation of this constraint is

$$
0=\left(\begin{array}{c}
t_{x} \\
t_{y} \\
t_{z}
\end{array}\right)(\underbrace{\left(\begin{array}{c}
f \hat{v} \\
-f \hat{u} \\
y \hat{u}-x \hat{v}
\end{array}\right)}_{=: M}-\underbrace{\left(\begin{array}{ccc}
-\left(f^{2}+y^{2}\right) & x y & f x \\
x y & -\left(f^{2}+x^{2}\right) & f y \\
f y & -\left(x^{2}+y^{2}\right)
\end{array}\right)}_{=: H}\left(\begin{array}{l}
r_{x} \\
r_{y} \\
r_{z}
\end{array}\right)),
$$

which Heeger and Jepson describe during the course of their subspace construction. In detail, they use a subspace which is orthogonal to the base polynomial defined by entries of the matrix $H\left(x_{i}, y_{i}\right)_{i=1 . . m}$, where $m$ denotes the finite number of constraints employed [9].

Optimization of translation. Only a linear independent part of the base polynomial $H$ is used for optimization. We chose the upper triangular matrix together with the diagonal of matrix $H$. These entries are summarized in the vector $E:=\left(-\left(f^{2}+y^{2}\right), x y, f x,-\left(f^{2}+x^{2}\right), f y,-\left(x^{2}+y^{2}\right)\right)^{t}$. To achieve a linear form of Equation 2 auxiliary variables $\left(t_{x} \cdot r_{x}, t_{x} \cdot r_{y}, t_{x} \cdot r_{z}, t_{y} \cdot r_{y}, t_{y} \cdot r_{z}, t_{z} \cdot r_{z}\right)^{t}:=K$ are introduced. With respect to $E$ and $K$ the linear optimization problem

$$
F(\hat{V} ; T, K(T)):=\int_{\Omega_{x}}\left[T^{t} M+K^{t} E\right]^{2} d \boldsymbol{x} \stackrel{T, K(T)}{\longrightarrow} \min ,
$$

is defined, integrating constraints over all locations $\boldsymbol{x}=(x, y) \in \Omega_{\boldsymbol{x}} \subset \Re^{2}$ of the image plane. This image plane is assumed to be continuous and finite. Calculating partial derivatives of $F(\hat{V} ; T, K(T))$ and equating them to zero leads 
to the linear system of equations

$$
\begin{aligned}
& 0=\int_{\Omega_{\boldsymbol{x}}}\left[T^{t} M+K^{t} E\right] \cdot E^{t} d \boldsymbol{x}, \\
& 0=\int_{\Omega_{\boldsymbol{x}}}\left[T^{t} M+K^{t} E\right] \cdot\left[M+\frac{\partial\left(K^{t} E\right)}{\partial T}\right]^{t} d \boldsymbol{x},
\end{aligned}
$$

consisting of nine equations and nine variables in $K$ and $T$. Solving Equation 4 with respect to $K$ and inserting the result as well as the partial derivative for the argument $T$ of expression $K$ into Equation 5 results in the homogenous linear system of equations

$$
\begin{aligned}
0 & =T^{t} \int_{\Omega_{\boldsymbol{x}}} L_{i} L_{j} d \boldsymbol{x}=: T^{t} C, \quad i, j=1 . .3, \quad \text { with } \\
L_{i} & :=M_{i}-(D E)^{t} \int_{\Omega_{\boldsymbol{x}}} E M_{i} d \boldsymbol{x}, \quad i=1 . .3 \text { and } D:=\left[\int_{\Omega_{\boldsymbol{x}}} E E^{t} d \boldsymbol{x}\right]^{-1} \in \Re^{6 \times 6} .
\end{aligned}
$$

A robust (non-trivial) solution for such a system is given by the eigenvector which corresponds to the smallest eigenvalue of the $3 \times 3$ scatter matrix $C$ [3].

Removal of statistical bias. All methods which are based on the bilinear constraint given in Equation 2 are statistically biased [9, 11, 14, 18]. To calculate this bias we define an isotropic noisy input by the vector $\tilde{V}:=(\hat{u}, \hat{v})+\left(n_{u}, n_{v}\right)$, with components $n_{u}$ and $n_{v} \in N(\mu=0, \sigma)$ normally distributed. A statistical bias is inferred by studying the expectation value $\langle\cdot>$ of the scatter matrix $\tilde{C}$. This scatter matrix is defined inserting the noisy input flow $\tilde{V}$ into Equation 6 . This gives $<\tilde{C}>=<C>+\sigma^{2} N$ with

$$
N=\left(\begin{array}{ccc}
f & 0 & -f<x> \\
0 & f & -f<y> \\
-f<x>-f<y>< & \left(x^{2}+y^{2}\right)>
\end{array}\right),
$$

using the properties $\left\langle n_{u}>=<n_{v}>=0\right.$ and $<n_{u}^{2}>=<n_{v}^{2}>=\sigma^{2}$. Several procedures to remove the bias term $\sigma^{2} N$ have been proposed. For example, Kanatani suggested a method of renormalization subtracting the bias term on the basis of an estimate of $\sigma^{2}[11]$. Heeger and Jepson used dithered constraint vectors and defined a roughly isotropic covariance matrix with these vectors. MacLean used a transformation of constraints into a space where the influence by noise is isotropic [14]. Here, the last approach is used, due to its computational efficiency. In a nutshell, to solve Equation 6 considering noisy input we calculate the eigenvector which corresponds to the smallest eigenvalue of matrix $\tilde{C}$. Prewithening of the scatter matrix $\tilde{C}$ gives $\check{C}:=N^{-\frac{1}{2}} \tilde{C} N^{-\frac{1}{2}}$. Then the influence by noise is isotropic, namely $\sigma^{2} I$, where $I$ denotes a $3 \times 3$ unity matrix. The newly defined eigenvalue problem $\check{C} x=\left(\lambda+\sigma^{2}\right) x$ preserves the ordering of $\lambda$ and eigenvectors $N^{-\frac{1}{2}} x$ compared to the former eigenvalue problem $C x=\lambda x$. Then the solution is constructed with the eigenvector of matrix $\check{C}$ which corresponds to the smallest eigenvalue. Finally, this eigenvector has to be multiplied by $N^{-\frac{1}{2}}$. 


\section{Results}

To test the proposed method for ego-motion estimation in different configurations we use two sequences, the Yosemite sequence ${ }^{1}$ and the Fountain sequence ${ }^{2}$. In the Yosemite sequence a flight through a valley is simulated, specified by $T=(0,0.17,0.98) \cdot 34.8 \mathrm{px}$ and $R=(1.33,9.31,1.62) \cdot 10^{-2} \mathrm{deg} /$ frame $[9]$. In the Fountain sequence the curvilinear motion with $T=(-0.6446,0.2179,2.4056)$ and $R=(-0.125,0.20,-0.125) \mathrm{deg} /$ frame is performed. The (virtual) camera employed to gather images has a vertical field of view of $40 \mathrm{deg}$ and a resolution of $316 \times 252$ for the Yosemite sequence and $320 \times 240$ for the Fountain sequence. All methods included in our investigation have a statistical bias which is removed with the technique of MacLean [14]. The iterative method of Pauwels and Van Hulle [18] employs a fix-point iteration mechanism using a maximal number of 500 iterations and 15 initial values for the translation direction, randomly distributed on the positive hemisphere [18].
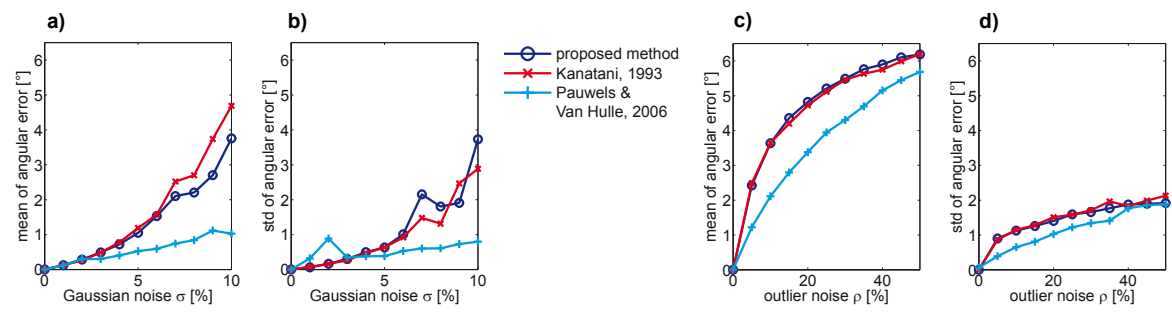

Fig. 1. All methods employed show numerical stability in the presence of noise due to small translational and rotational errors (not shown). In detail, a) shows the mean angular error for Gaussian noise and c) for outlier noise. Graphs in b) and d) show the corresponding standard deviation, respectively. The parameter $\sigma$ is specified with respect to the image height. Mean and standard deviation are calculated for a number of 50 trials.

Numerical stability. To show numerical stability we use the scenic depth of the Fountain sequence (5th frame) with a quarter of the full resolution to test different ego-motions. These ego-motions are uniformly distributed in the range of $\pm 40 \mathrm{deg}$ azimuth and elevation in the positive hemisphere. Rotational components for pitch and yaw are calculated by fixating the central point and compensating translation by rotation. An additional roll component of $1 \mathrm{deg} /$ frame is superimposed. With scenic depth values and ego-motion given, optical flow is calculated by Equation 1. This optical flow is systematically manipulated by applying two different noise models: a Gaussian and an outlier noise model. The Gaussian noise model was former specified in Section 3. In the outlier noise

\footnotetext{
${ }^{1}$ Available via anonymous ftp from ftp.csd.uwo.ca in the directory pub/vision.

${ }^{2}$ Provided at http://www.informatik.uni-ulm.de/ni/mitarbeiter/FRaudies.
} 
model a percentage, denoted by $\rho$, of all flow vectors are replaced by a randomly constructed vector. Each component of this vector is drawn from a uniformly distributed random variable. The interval of this distribution is defined by the negative and positive of the mean length of all flow vectors. Outlier noise models sparsely distributed gross errors, e.g. caused by correspondences that were incorrectly estimated. Applying a noise model to the input flow, the estimation of ego-motion becomes erroneous. These errors are reported by: (i) the angular difference between two translational 3-d vectors, whereas one is the estimated vector and the other the ground-truth vector, and (ii) the absolute value of the difference for each rotational component. Again, differences are calculated between estimate and ground-truth. Figure 1 shows errors of ego-motion estimation applying the Gaussian and the outlier noise model. All methods show numerical stability, whereas the mean translational error is lower than approximately 6 deg for both noise models. The method of Pauwels and Van Hulle performs best compared to the other methods. Better performance is assumed to be achieved by employing numerical fix-point iteration with different initial values randomly chosen within the search space.

Table 1. Errors for estimated optical and ground-truth input flow of the proposed method. In case of the Yosemite sequence which contains the independently moving cloudy sky the RANSAC paradigm is employed which improves ego-motion estimates (50 trials, mean and \pm standard deviation shown). ${ }^{(x)} \varangle$ denotes the angle calculated between estimated and ground-truth 3 -d translational vectors.

\begin{tabular}{|c|c|c|c|c|}
\hline sequence & $\begin{array}{c}\text { translation } \\
\varangle\left(T^{e s t}, T^{g t}\right)^{(x)}[\mathrm{deg}]\end{array}$ & $\left|\Delta r_{x}\right|[\mathrm{deg}]$ & $\begin{array}{c}\text { rotation } \\
\left|\Delta r_{y}\right|[\mathrm{deg}]\end{array}$ & $\left|\Delta r_{z}\right|[\mathrm{deg}]$ \\
\hline \multicolumn{5}{|c|}{ estimated optical flow; Brox et al. [2]; 100\% density } \\
\hline Fountain & 4.395 & 0.001645 & 0.0286 & 0.02101 \\
\hline Yosemite & 4.893 & 0.02012 & 0.1187 & 0.1153 \\
\hline \multicolumn{5}{|c|}{ estimated optical flow; Farnebaeck [6]; 100\% density } \\
\hline Fountain & 6.841 & 0.01521 & 0.05089 & 0.025 \\
\hline Yosemite & 4.834 & 0.03922 & 0.00393 & 0.07636 \\
\hline \multicolumn{5}{|c|}{ estimated optical flow; Farnebeack, [6];25\% density } \\
\hline$\overline{\text { Fountain }}$ & 1.542 & 0.0008952 & 0.01349 & 0.003637 \\
\hline Yosemite & 1.208 & 0.007888 & 0.01178 & 0.02633 \\
\hline \multirow[t]{2}{*}{ Yosemite (RANSAC) } & 1.134 & 0.01261 & 0.008485 & 0.02849 \\
\hline & \pm 0.2618 & & & \pm 0.003714 \\
\hline \multicolumn{5}{|c|}{$25 \%$ of full resolution } \\
\hline$\overline{\text { Fountain }}$ & $\overline{0.0676}$ & 0.000259 & $8.624 \mathrm{e}-006$ & 0.0007189 \\
\hline Yosemite & 5.625 & 0.02613 & 0.1092 & 0.06062 \\
\hline \multirow[t]{2}{*}{ Yosemite (RANSAC) } & 1.116 & 0.01075 & 0.004865 & 0.02256 \\
\hline & \pm 1.119 & \pm 0.01021 & \pm 0.006396 & \pm 0.009565 \\
\hline
\end{tabular}

Estimated optical flow as input. We test our method on the basis of optical input flow estimated by two different methods. 
First, we utilize the tensor-based method of Farnebaeck together with an affine motion model [6] to estimate optical flow. The spatio-temporal tensor is constructed by projecting the input signal to a set of base polynomials of finite Gaussian support ( $\sigma=1.6 \mathrm{px}$ and length $l=11 \mathrm{px}, \gamma=1 / 256)$. Spatial averaging of the resulting components of the tensor is performed with a Gaussian filter $(\sigma=6.5 \mathrm{px}$ and $l=41 \mathrm{px})$.

Second, optical flow is estimated with the affine warping technique of Brox et al. [2]. Here, we implemented the 2-d version of the algorithm and used the following parameter values, $\alpha=200, \gamma=100, \epsilon=0.001, \sigma=0.5, \eta=0.95$, a number of 77 outer fix point iterations and 10 inner fix point iterations. To solve partial differential equations the numerical method Successive Over-Relaxation (SOR) with parameter $\omega=1.8$ and 5 iterations is applied.

Errors of optical flow estimation are reported by a 3-d mean angular error which was defined by Barron and Fleet [1]. According to this angular error optical flow is estimated for frame pair $8-9$ (starting to count from index 0 ) of the Yosemite sequence, with $5.41 \mathrm{deg}$ accuracy for the method of Farnebaeck and with $3.54 \mathrm{deg}$ for the method of Brox. In case of frame pair $5-6$ of the Fountain sequence the mean angular error is $2.49 \mathrm{deg}$ estimating flow with Farnebaeck's method and $2.54 \mathrm{deg}$ for the method of Brox. All errors refer to a density of $100 \%$ for optical flow data.

Table 1 lists errors of ego-motion estimation for different scenarios. Comparing the first two parts of the table, we conclude that a high accuracy for optical flow estimates does not necessarily provide a high accuracy in the estimation of ego-motion. In detail, the error of ego-motion estimation depends on the error characteristic (spatial distribution and value of errors) within the estimated optical flow field. However, this characteristic is not expressed by the mean angular error. One way to reduce the dependency on the error characteristic is to reduce the data set, leaving out data points which are most erroneous. Generally, this requires (i) an appropriate confidence measure to evaluate the validity or reliability of data points, (ii) and a strategy to avoid linear dependency in the resulting data w.r.t. ego-motion estimation. Farnebaeck describes how to calculate a confidence value within his thesis [6]. Here, this confidence is used to thin out flow estimates, whereas we retain $25 \%$ of all estimates, enough to avoid linear dependency for our configurations. For ego-motion estimation errors are then reduced as can be observed in the third part of Table 1. In case of the Yosemite sequence sparsification has a helpful side effect. The cloud motion is estimated by the method of Farnebaeck with low accuracy and confidence. Thus, no estimates corresponding from the cloudy sky are contained in the data set for the estimation of ego-motion. In the last part of Table 1 ground-truth optical flow is utilized to estimate ego-motion. In this case, the cloudy sky is present in the data set and thus deflects estimates of ego-motion, e.g. the translational angular error amounts $5.6 \mathrm{deg}$. To handle IMOs we use the RANSAC algorithm [7]. In a nutshell, the idea of the algorithm is to achieve an estimate which is based on non erroneous data points only. Therefore, initial estimates are performed on different randomly selected subsets of all data points, which 
are tried to be enlarged by other non erroneous data points. The algorithm stops if an estimate is found, that is based on a data set of a certain cardinality. For the ground-truth flow of the Yosemite sequence, this method is successfully in estimating ego-motion, now the translational angular error amounts $1.116 \mathrm{deg}$ (mean value).

\section{Discussion}

A novel linear optimization method was derived to solve the segregation of the translational and rotational component, one of the main problems in computational ego-motion estimation $[13,3,9]$.

Related work. A well-known linear method for ego-motion estimation is the subspace method [9]. Unlike our method a subspace independent of the rotational part was used by Heeger and Jepson for the estimation of translation, using only $m-6$ of $m$ constraints. However, in the method proposed here, all constraints are used which leads to more robust estimates. Zhuang et al. formulated a linear method for the segregation of translation and rotation employing the instantaneous motion model together with the epipolar constraint [21]. They introduced auxiliary variables, as superposition of translation and rotation, then optimized w.r.t. these variables and translation. In a last step they reconstructed rotation from auxiliary variables. Unlike their method we used the bilinear constraint for optimization, defined auxiliary variables differently, split up the optimization for rotation and translation and finally had to solve only a $3 \times 3$ eigenvalue problem for translation estimation, instead of a $9 \times 9$ eigenvalue problem in case of Zhuang's approach. Moreover, applying this different optimization strategy allowed us to incorporate the method of MacLean to remove a statistically bias, which is not the case for the method of Zhuang.

Table 2. Average (1000 trials) computing times [msec] of methods estimating egomotion, tested with a $\mathrm{C}++$ implementation on a Windows XP platform, Intel Core 2 Duo T9300. ${ }^{(*)}$ This algorithm employs a maximal number of 500 iterations and 15 initial values.

\begin{tabular}{|c|c|c|c|c|}
\hline \multirow[t]{2}{*}{ method } & \multicolumn{4}{|c|}{ number of vectors } \\
\hline & $25 \quad 225$ & 2.025 & 20.164 & 80.089 \\
\hline$\overline{\text { new proposed } n}$ & 0.050 .06 & 0.34 & 4.56 & $\overline{22.16}$ \\
\hline Kanatani (unbiased) & 0.030 .11 & 0.78 & 7.56 & 29.20 \\
\hline Heeger \& Jepson (unbiased) & 0.082 .44 & 399.20 & n.a. & n.a. \\
\hline Pauwels \& Van Hulle, 2006 (unbiased) & 0.160 .81 & 6.90 & 66.87 & 272.95 \\
\hline
\end{tabular}

Complexity. To achieve real-time capability in applications a low computationally complexity is of vital need. Existing methods for the estimation of ego-motion have a higher complexity than our method (compare with Table 2). For example [9] employs a singular value decomposition for a $m \times 6$ matrix, 
or iterative methods to solve for nonlinear optimization problems are employed $[4,18,20]$. Comparable to our method in case of computational complexity, is the method of Kanatani [11]. Unlike our approach this method is based on the epipolar constraint.

Numerical stability. We showed that the optimization method is robust against noise, compared to other ego-motion algorithms [11,18]. Furthermore, the technique of pre-whitening is applied to our method to remove a statistical bias as well. This technique was proposed by MacLean [14] for bias removal in the subspace algorithm of Heeger and Jepson [9] and the method by Pauwels and Van Hulle for the fix-point iteration, iterating between coupled estimates for translation and rotation of ego-motion [17]. Unlike other unbiasing techniques MacLean's technique needs neither an estimate of the noise characteristic nor an iterative mechanism. With statistical bias removed methods are consistent in the sense of Zhang and Tomasi's definition of consistency [20].

Outlier detection. To detect outliers in ego-motion estimation, in particular IMOs, several methods were suggested, namely frameworks employing the EM algorithm [15, 5], the Collinear Point Constraint [12] and the RANSAC algorithm [19]. In accordance to the conclusion of Torr's thesis, who found that the RANSAC algorithm performs best in motion segmentation and outlier detection, we chose RANSAC to achieve robust ego-motion estimation.

\section{Conclusion}

In summary, we have introduced a novel method for the separation of translation and rotation in the computation of ego-motion. Due to the simplicity of the method it has a very low computational complexity and is thus faster than existing estimation techniques (Table 2). First, we tested our method with a computed optical flow field, where ego-motion can be estimated exactly. Under noisy conditions results show numerical stability of the optimization method and its comparability with existing methods for the estimation of ego-motion. In more realistic scenarios utilizing estimated optical flow, ego-motion can be estimated with high accuracy. Future work will employ temporal integration of ego-motion estimates within the processing of an image sequences. This should stabilize ego-motion and optical flow estimation counting on the spatio-temporal coherence of the visually observable world.

\section{Acknowledgements}

Stefan Ringbauer kindly provided a computer graphics ray-tracer utilized to generate images and ground-truth flow for the Fountain sequence. This research has been supported by a scholarship given to F.R. from the Graduate School of Mathematical Analysis of Evolution, Information and Complexity at Ulm University. 


\section{References}

1. J.L. Barron, D.J. Fleet, and S.S. Beauchemin. Performance of optical flow techniques. Int. J. of Comp. Vis., 12(1):43-77, 1994.

2. T. Brox, A. Bruhn, N. Papenberg, and J. Weickert. High accuracy optical flow estimation based on a theory for warping. In T. Pajdla and J. Matas, editors, Proc. Europ. Conf. on Comp. Vis., LNCS 3024, pages 25-36, 2004.

3. A.R. Bruss and B.K.P. Horn. Passive navigation. Comp. Vis., Graph., and Im. Proc., 21:3-20, 1983.

4. A. Chiuso, R. Brockett, and S. Soatto. Optimal structure from motion: Local ambiguities and global estimates. Int. J. of Comp. Vis., 39(3):195-228, 2000.

5. M. Clauss, P. Bayerl, and H. Neumann. Segmentation of independently moving objects using a maximum-likelihood principle. In R Lafrenz V Avrutin P Levi, M Schanz, editor, Autonome Mobile Systeme 2005, Informatik Aktuell, Springer, Berlin, pages 81-87, 2005.

6. G. Farnebaeck. Polynomial expansion for orientation and motion estimation. PhD thesis, Dept. of Electrical Engineering, Linkoepings universitet, 2002.

7. M.A. Fischler and R.C. Bolles. Random sample consensus: A paradigm for model fitting with applications to image analysis and automated cartography. Comm. of the ACM, 24(6):381-395, 1981.

8. J.J. Gibson. The Perception of the Visual World. Houghton Mifflin, Boston, 1950.

9. D.J. Heeger and A.D. Jepson. Subspace methods for recovering rigid motion i: Algorithm and implementation. Int. J. of Comp. Vis., 7(2):95-117, 1992.

10. H. Helmholtz. Treatise on physiological optics. ed. J.P.Southhall, 1925.

11. K. Kanatani. 3-d interpretation of optical-flow by renormalization. Int. J. of Comp. Vis., 11(3):267-282, 1993.

12. N.V. Lobo and J.K. Tsotsos. Computing ego-motion and detecting independent motion from image motion using collinear points. Comp. Vis. and Img. Underst., 64(1):21-52, 1996.

13. H.C. Longuet-Higgins and K. Prazdny. The interpretation of a moving retinal image. Proc. of the Royal Soc. of London. Series B, Biol. Sci., 208(1173):385-397, 1980.

14. W.J. MacLean. Removal of translation bias when using subspace methods. In IEEE Int. Conf. on Comp. Vis., volume 2, pages 753-758, 1999.

15. W.J. MacLean, A.D. Jepson, and R.C. Frecker. Recovery of egomotion and segmentation of independent object motion using the EM algorithm. Brit. Mach. Vis. Conf., 1:175-184, 1994.

16. K. Pauwels and M.M. Van Hulle. Segmenting independently moving objects from egomotion flow fields. Proc. of the Early Cognitive Vision Workshop (ECOVISION 04), Isle of Skye, Scotland, 2004.

17. K. Pauwels and M.M. Van Hulle. Robust instantaneous rigid motion estimation. In Proc. of Comp. Vis. and Pat. Rec., volume 2, pages 980-985, 2005.

18. K. Pauwels and M.M. Van Hulle. Optimal instantaneous rigid motion estimation insensitive to local minima. Comp. Vis. and Im. Underst., 104(1):77-86, 2006.

19. P.H.S. Torr. Outlier Detection and Motion Segmentation. PhD thesis, Engineering Dept., University of Oxford, 1995.

20. T. Zhang and C. Tomasi. Fast, robust, and consistent camera motion estimation. In Proc. of Comp. Vis. and Pat. Rec., volume 1, pages 164-170, 1999.

21. X. Zhuang, T.S. Huang, N. Ahuja, and R.M. Haralick. A simplified linear optic flow-motion algorithm. Comp. Graph. and Img. Proc., 42:334-344, 1988. 